\title{
RHEOLOGY OF CARBOXYMETHYL CELLULOSE SOLUTIONS TREATED WITH CELLULASES
}

\author{
Jung Myoung Lee ${ }^{\text {a }}$, John A. Heitmann ${ }^{\text {a }}$, and Joel J. Pawlak ${ }^{a^{*}}$ \\ The effect of cellulase treatments on the rheology of carboxymethyl \\ cellulose $(\mathrm{CMC})$ solutions was studied using a rotational viscometer. \\ The rheological behaviors of CMC solutions of different molecular mass \\ and degrees of substitution where studied as a function of time after \\ various treatments. These solutions were subjected to active and heat- \\ denatured cellulase, a cationic polyelectrolyte (C-PAM), as well as \\ different shear rates. A complex protein-polymer interaction was \\ observed, leading to a potential error source in the measurement of \\ enzymatic activity by changes in the intrinsic viscosity. The interaction \\ was termed a polymeric effect and defined as a reduction in viscosity of \\ the substrate solution without significant formation of reducing sugars \\ from enzymatic hydrolysis. The cause of the reduction in viscosity \\ appears to be related to the interaction between the enzymes as \\ amphipathic particles and the soluble CMC. Thus, the polymeric effect \\ may cause a considerable experimental error in the measurement of \\ enzymatic activity by viscometric methods.
}

Keywords: Carboxymethyl cellulose (CMC), Endoglucanase activity, Cellulase, Viscosity, Rotational viscometer, Thermal denaturation, Polymeric effects

Contact information: a: Department of Wood and Paper Science, North Carolina State University, Box 8005, Raleigh, USA; *Corresponding author: jipawlak@ncsu.edu

\section{INTRODUCTION}

Cellulases are produced by a variety of bacteria and fungi. These enzymes have been used in a number of industrial applications in the fields of food science, textiles, and pulp and paper (Bhat 2000). These enzymes act on cellulosic substrates, both soluble and insoluble. Native cellulose has a complex ultrastructure with both crystalline and amorphous regions. The simultaneous synergistic action of several different isozymes of cellulase is required to complete the hydrolysis of solid cellulose. Based on their activity on cellulose, cellulases can be classified as endoglucanases (EGs), cellobiohydrases (CBHs), and $\beta$-glucosidases (Belgiun and Aubert 1994). EGs preferentially hydrolyze the $\beta$-(1-4) linkages of amorphous cellulose in a random manner. CBHs hydrolyze crystalline cellulose by cleaving the $\beta$-(1-4) linkage of the cellulose to produce cellobiose and may work synergistically with EG to depolymerize the cellulose. The cellobiose released from EG and $\mathrm{CBH}$ actions is finally converted into glucose by action of $\beta$ glucosidase. Thus, the structure-function relationship between the cellulase isozymes and the cellulosic substrate enhances our knowledge of cellulose biodegradation (Boisset et al. 1998). 
Enzyme activity is measured by the production of sugar reducing end group, which is taken to be an indication of cleavage of the cellulose molecules. It is also an indication of the efficiency of the cellulase hydrolyzing reaction. The determination of cellulase activity is a complicated process, because the hydrolysis of insoluble cellulose may not be linear with enzyme dosage and/or reaction time. Thus, the dosage of enzyme and the point in the reaction at which the activity is measured is critical. For such reasons, enzymatic activity should be carefully determined. Several standard substrates are available for the determination of cellulase activity in terms of the overall and EG activity (Ghose 1987). Filter papers have been used as a standard substrate to measure the total cellulase activity (Wu et al. 2006). The filter paper activity, termed as FPase, expressed the summations of the simultaneous synergistic action of EGs, CBHs, and $\beta$-glucosidase in a cellulase preparation. Soluble cellulose derivatives, such as carboxymethyl cellulose (CMC) and hydroxyethyl cellulose (HEC), may be used as substrates for determining EG activity. These substrates are rather specific to EG activity, since CBHs are not generally able to degrade substituted cellulose (Rouvinen et al. 1990). The hydrolysis rate of EG was conventionally determined by reduction of viscosity or by increase of reducing sugar end groups of the cellulose derivatives (Sharrock 1988; Mullings 1985). Recently, based on adsorption behaviors of free and bound enzymes on microcrystalline cellulose (MCC), a new measurement of EG activity has been introduced (Zhou et al. 2004). Among these methods, monitoring the reduction in CMC solution viscosity is considered to be the most accurate method to detect EG activity (Almin and Eriksson 1967; Valsenko et al. 1998). However, the viscometric method for measuring activity is not commonly used due to its laborious and discontinuous nature. For this reason, the colorimetric measurement of reducing sugars has been routinely used for measurement of EG activity.

Various types of automated viscometers are available to measure the rheological changes of a polymer solution. Viscosity is a fundamental rheological parameter that characterizes the resistance of the fluid to flow. The viscosity of a polymer solution is related to the polymer concentration, the extent of polymer-solvent interaction, and the polymer structure such as molecular weight, shape, molecular flexibility, and molecular conformation. Under appropriate experimental conditions, it is functionally related to the molecular weight (Browning 1967). However, a more complete understanding of the rheological changes in a cellulase-polymer solution is required to accurately monitor cellulose hydrolysis by viscosity measurements.

Like all proteins, enzymes are basically made up of amino acids linked by peptide bonds between the carboxyl groups of one amino acid and the amino group of the next amino acid. The hydrophobic and hydrophilic nature of amino acids often makes the surface of enzymes have an amphipathic interfacial structure, i.e. proteins tend to adsorb at interfaces as a surface-active polymer (Britt et al. 2003). Moreover, CMC is a semiflexible anionic polymer (Hoogendam et al. 1998). The adsorption of enzymes (protein) on the CMC leads to a change of the polymer behavior. Of particular interest is the effect of enzyme absorption on the rheology of a cellulase-CMC solution. Mixtures of proteins (enzymes) and polymers in aqueous dispersion are often accompanied by either segregative or associative phase separation (Doublier et al. 2000). Thus, physical and chemical parameters of the proteins (enzymes) and substrate and solution characteristics such as solution $\mathrm{pH}$, ionic strength, concentration and protein/polymer 
ratio should be considered. Moreover, processing variables such as temperature, shear rate, and time also strongly influence the rheological behavior of the complexes. For example, changes in functional properties due to soluble complexes between a globular protein (BSA) and a polysaccharide (CMC) have been reported (Tuiner et al. 2000; Renard et al. 1997). The protein was adsorbed onto the CMC segments in the dilute regime or entrapped in the polymer network in the semi-dilute region. After thermal treatment of the soluble complexes, a considerable change in the visco-elastic properties of the network was observed (Renard et al. 1998).

When a cellulase is added to a soluble cellulose derivative (e.g. CMC), one might expect that the rheological behavior of the mixture will change considerably as a function of reaction time. The change in the viscosity may be due to the cellulase ad- and desorbing or entrapping the substrate. The configuration and conformation of the substrate molecules will then be changed. These interactions between cellulases and substrates may be expected to result in considerable changes in the solution viscosity. Thus, significant changes in the solution viscosity may result without any enzymatic hydrolysis taking place. It should also be noted that reductions of enzymatic activity and its binding ability are expected when enzymes are continuously subjected to shear or exposed to an air-liquid interface during mixing and agitation processes (Kaya et al. 1994; Kim et al. 1982).

The study reported herein investigates the rheological behavior of CMC solutions during enzyme hydrolysis and the influence of enzymes on CMC solutions without hydrolysis. The ability of enzymes to reduce $\mathrm{CMC}$ solution viscosity without hydrolysis was investigated in two manners. First a heat-denatured enzyme was applied to the CMC solution. Second, a high degree of substitution CMC was used. This CMC could not be degraded by the enzymes. In addition, the effects of shear rate and contact time were evaluated during the cellulase-polymer interaction.

\section{EXPERIMENTAL}

\section{Materials}

A commercial enzyme preparation supplied by Dyadic International (Fibrezyme L, Florida, USA) was used in this study. The cellulase preparation was fractionated by using a $10 \mathrm{kD}$ cut-off filter (Amicon, USA) and then used as an active cellulase preparation. In order to make a heat-denatured cellulase preparation, a $10 \mathrm{~g}^{-1}$ solution of the active cellulase preparation was transferred to a glass tube and covered. The tube was then placed in a boiling water bath for 2 hours with vigorous agitation. After the boiling, the suspension was homogenized for 15 minutes with an ultrasonic homogenizer (Omni Ruptor 250, Omni international Inc, USA) in order to make a well dispersed heatdenatured cellulase suspension. The active and heat-denatured cellulase suspensions were refrigerated during storage.

Five commercial carboxymethyl celluloses (CMCs) in the sodium salt form of different average degrees of polymerization (DP) and degrees of substitution (DS) were obtained from Aqualon Hercules Inc. (Wilmington, Delaware, USA): 7L (0.7 DS and 400 DP), 7M (0.7 DS and 1,100 DP), 7H (0.7 DS and 3,200 DP), 9M8 (0.9 and 800 DP), and 
12M8 (1.2 DS and $800 \mathrm{DP})$. The letters L, M, and H correspond to low, medium, and high molecular mass. According to the manufacturer, the typical molecular mass values corresponding to these categories are 90,000, 250,000, and 700,000 Daltons. A cationic polyacrylamide (C-PAM) (Percol ${ }^{\circledR} 175$, from Ciba Specialty Chemicals) was also used. Its molecular mass is approximately 5,000,000 Daltons.

\section{Methods}

\section{Preparation of CMC solutions}

An appropriate amount of the $\mathrm{CMCs}$ was slowly added into a sodium acetate buffer $(50 \mathrm{mM}, \mathrm{pH} 4.7)$ to make $1.0 \%$ and $1.4 \%$ concentrations of CMC suspensions under vigorous agitation. After completing the addition, the suspensions were transferred to a blender and vigorously mixed for one minute at room temperature. The blended suspension was stirred for one hour, while heating to $80{ }^{\circ} \mathrm{C}$. In order to make homogenous CMC solutions, the suspension was then filtered through a sintered glass (coarse) funnel into a vacuum filtering flask. The suspension was then agitated for one hour at room temperature under the vacuum to remove entrapped air bubbles. The CMC solutions were stored at $4{ }^{\circ} \mathrm{C}$ and used within one week. After one week, fresh $\mathrm{CMC}$ solutions were again prepared.

Enzymatic hydrolysis and its viscosity measurement

Enzymatic hydrolysis of CMC solutions was performed using a Brookfield rotational viscometer with a UL adaptor (Brookfield DV-II+2, USA). $20 \mathrm{ml}$ of each $\mathrm{CMC}$ suspension was preheated at $50{ }^{\circ} \mathrm{C}$ in a water bath before being transferred to the UL adaptor, which was controlled to $50{ }^{\circ} \mathrm{C}$. One $\mathrm{ml}$ of each cellulase preparation was added to the CMC suspension to start the enzymatic reaction. The apparent viscosity of

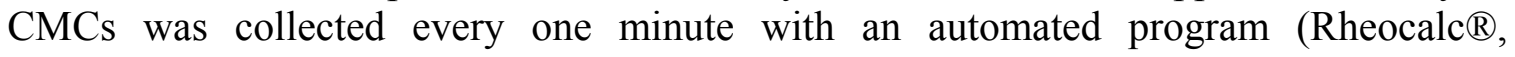
Brookfield instrument, USA). The viscometer used a rotational speed of $10 \mathrm{rpm}$ except during the shear rate dependence experiments. During this set of experiments the shear rates were varied in the range of 1 to $32 \mathrm{rpm}$.

\section{Reducing sugar measurement}

The reaction products of enzymatic hydrolysis were transferred into a test tube as quickly as possible to determine the concentration of reducing sugar end-groups in the sample. The amounts of reducing sugar end groups formed by cellulase action were determined by the dinitrosalicylic acid (DNS) method (Miller et al. 1960) using glucose to generate a standard curve.

\section{RESULTS AND DISCUSSION}

\section{Polymeric and Enzymatic Effects of Cellulase}

Figure 1 shows the change of the apparent viscosity as a function of time with the addition of active and heat-denatured enzymes. It should be noted that the zero time viscosity of the CMC solutions are indicative of the pure CMC solution viscosity. Also, 
at the enzyme concentration used, there was no detectable difference between water and the enzyme solution viscosity. It is clear from this figure that both the active and the heat-denatured enzyme reduce the viscosity of the CMC solution as a function of contact time. This seems to imply that the heat-denatured enzyme preparation retains a portion of its activity, although the preparation was brought to boiling for 2 hours.

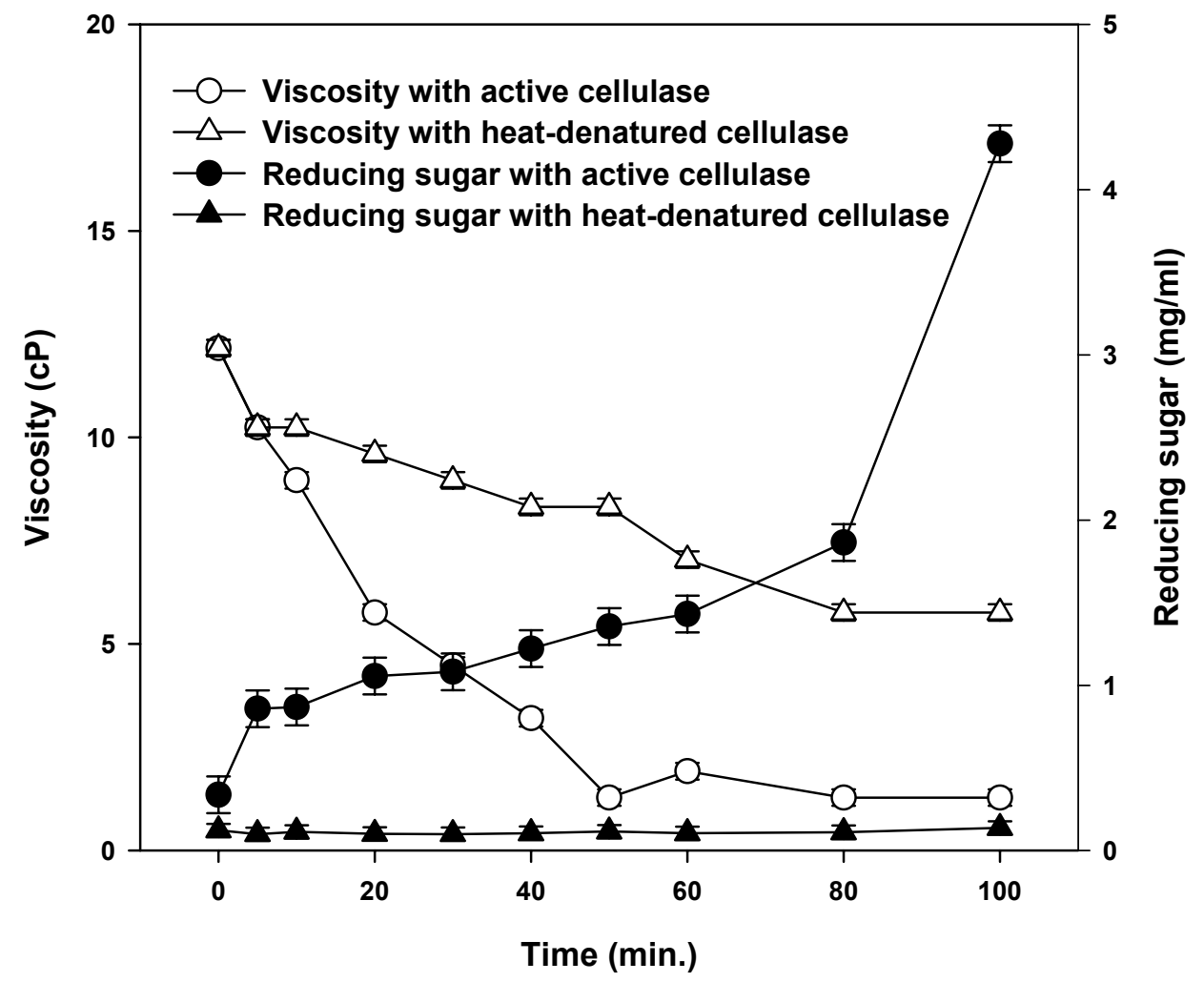

Fig. 1. Changes in apparent viscosity $\left(50^{\circ} \mathrm{C}\right.$ and $\left.10 \mathrm{rpm}\right)$ and reducing sugar of $7 \mathrm{M} \mathrm{CMC}(1.0 \%$ conc.) treated with active and heat-denatured cellulase as a function of time.

In order to examine whether the change in viscosity of the solution with the heatdenatured enzyme is from its residual activity, the production of reducing end groups in the hydrolysates from the active and heat-denatured cellulases were analyzed by the DNS method. The concentration of reducing sugar (glucose equivalent) liberated from the $\mathrm{CMC}$ can be used as a measure of hydrolytic efficiency of the active and heat-denatured cellulase preparations. The active cellulase depolymerized the substrate as a function of reaction time, leading to increased formation of the reducing sugar, whereas the heatdenatured preparation showed no significant change in the reducing sugar. It appears likely that the thermal denaturation of cellulases (Baker et al. 1992; Andreaus et al. 1999) and proteins (Kelly et al. 1994; Shimada and Cheftel 1989) caused partial unfolding of the proteins due to the disruption of the hydrogen bonds responsible for the threedimensional structure. This may expose highly reactive groups buried inside the protein structure to the solution. This may lead to increased sites available for the CMC to interact with the de-natured enzyme causing conformational changes of the substrate (Kelly et al. 1994). Based on this observation, the results in Figure 1 imply that the heat- 
denatured enzyme alters the conformation of the CMC and thus changes the viscosity of the solution. The change in the viscosity due to the amphipathic nature of the enzyme has been the termed the polymeric effect in this study.

\section{Effect of Cationic Polymer}

A decrease in the viscosity of a CMC solution was also observed when a cationic polymer was added to the solution, cf. Figure 2 . In this figure, one can see a decrease in the $\mathrm{CMC}$ solution viscosity over time with cationic polymer and heat-denatured enzyme addition. After 90 minutes of elapsed time the solutions were left in the viscometer for 500 minutes without shearing, and then the viscosities of the solutions were again measured. The viscosity with heat-denatured enzyme decreased slightly ( $3 \mathrm{cPs})$, whereas the viscosity with the cationic polymer increased over $500 \mathrm{cPs}$. This indicates that the cationic polyelectrolyte (C-PAM) interacts with the anionic polymer (CMC) as a function of time, forming a complex, and thus leading to an increase in the viscosity. This increase, in the absence of stirring, may be attributed to the high degree of polymerization of the C-PAM.

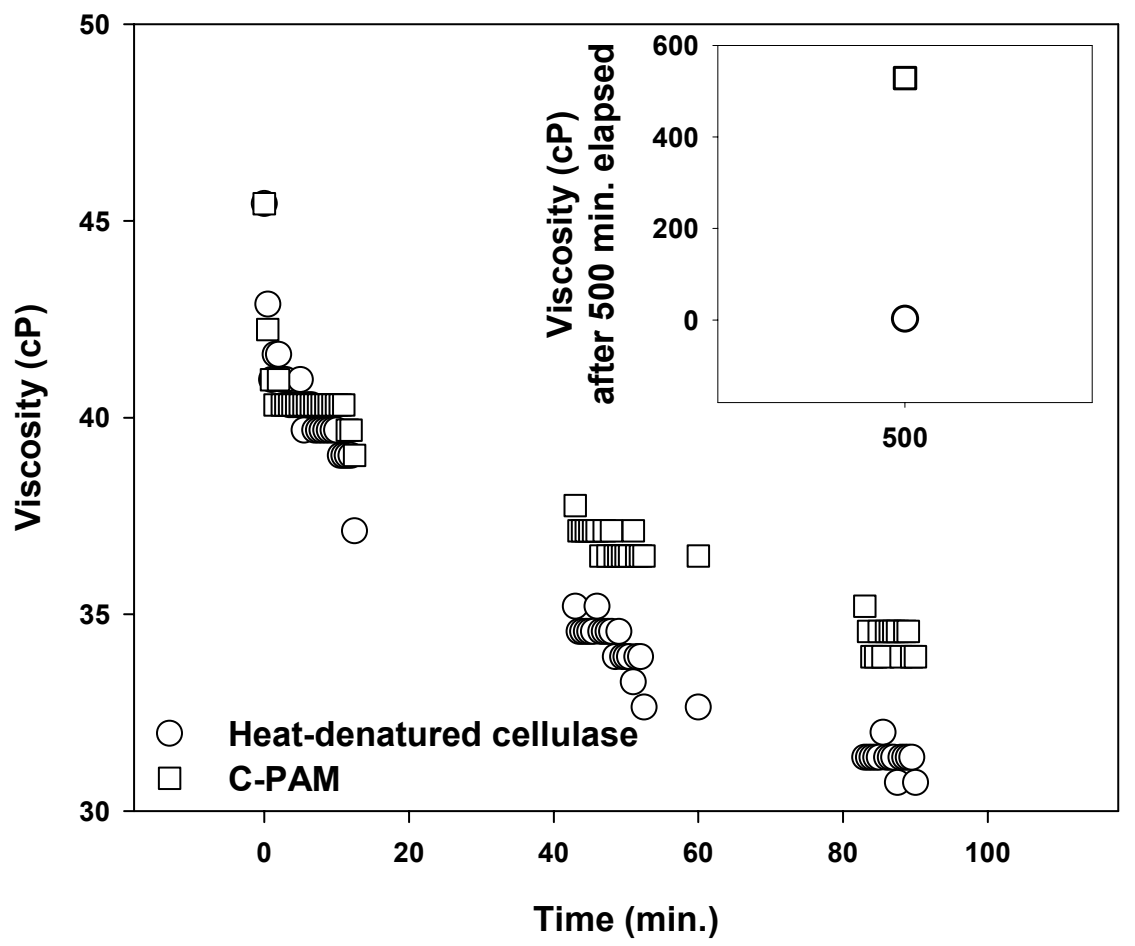

Fig. 2. Changes in apparent viscosity of $7 \mathrm{M} \mathrm{CMC} \mathrm{(1.4 \%} \mathrm{conc.)} \mathrm{treated} \mathrm{with} \mathrm{the} \mathrm{heat-denatured}$ enzyme or C-PAM $\left(0.1 \%\right.$ conc.) at $50{ }^{\circ} \mathrm{C}$ and $10 \mathrm{rpm}$ as a function of time. The inserted plot represents the apparent viscosity of the CMC after 500 minutes elapsed.

\section{Effect of CMCs with Different DS}

The polymeric effect, which reduces the CMC viscosity without the creation of reducing end groups, can also be observed using CMC with different degrees of substitution. Figure 3 shows the viscosity of CMC with different degrees of substitution 
as a function of enzyme contact time. Also shown in this figure is the concentration of reducing end groups as a function of time. It is known that the susceptibility of CMC to enzyme hydrolysis is dependent on degree of substitution (DS) of the CMC. When the DS of CMC reaches an average of one substitution per glucose unit, steric factors strongly retard the enzyme activity (Focher et al. 1991). Thus the International Union of Pure and Applied Chemistry (IUPAC) recommended CMC having a DS of 0.7 as a substrate for measuring cellulase activity (Ghose 1987). The inhibition of enzyme activity at high DS is shown again here by the lack of increase in reducing end group concentration with increased reaction time for the 1.2 DS CMC. However, it is noted that the viscosities of $\mathrm{CMC}$ decreased in a similar manner for all degrees of substitution, regardless of the change in the reducing end groups. This again indicates that a "polymeric effect" plays an important role in determining the viscosity of the CMC solution independent of hydrolytic activity.

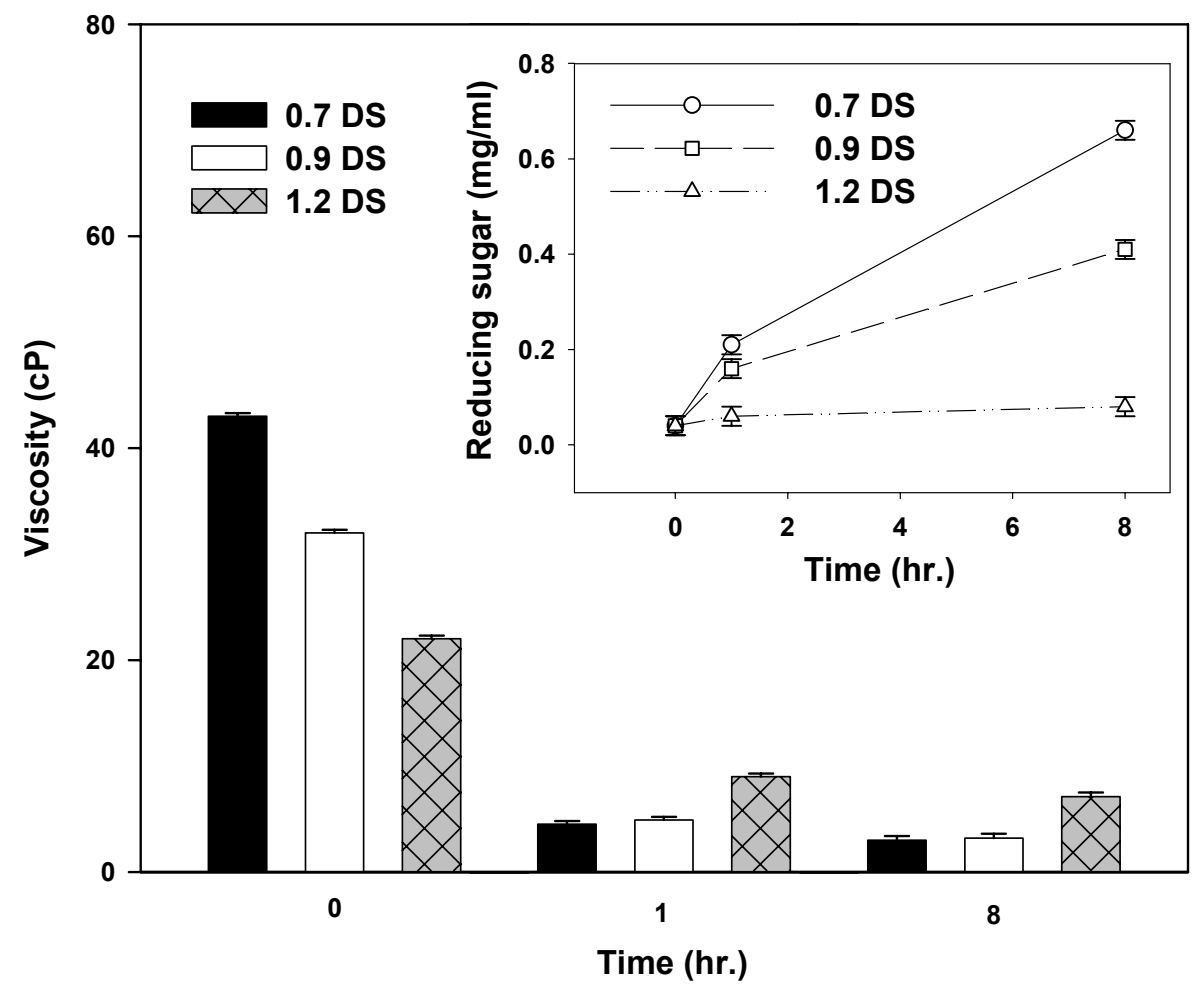

Fig. 3. Changes of apparent viscosity and reducing sugar of $7 \mathrm{M}, 9 \mathrm{M}$, and $12 \mathrm{M} \mathrm{CMCs}(1.4 \%$ conc.) treated with the active cellulase.

\section{Changes of Intrinsic Viscosity}

When measuring the enzymatic activity via changes in solution viscosity, the polymeric effect can result in significant errors in the measured activity, as shown in Figures 1-3. To evaluate the impact of the polymeric effect on the activity measurement, one needs to observe the intrinsic viscosity as a function of time, as the intrinsic viscosity is related to the molecular weight of the polymer. The intrinsic viscosity of a polymer solution is determined by measuring apparent viscosity at a series of different solute 
concentrations $(0.5,1.0,2.0$, and $3.0 \%$ with the $7 \mathrm{M} \mathrm{CMC})$. The intrinsic viscosity was evaluated from the extrapolation to zero concentration of $\ln \eta_{\mathrm{rel}} / \mathrm{C}$ against concentration C, using the Kraemer equation (Kraemer 1938):

$$
\frac{\ln \eta_{r e l}}{C}=[\eta]+\kappa^{\prime \prime}[\eta]^{2} C
$$

where $[\eta]$ is intrinsic viscosity, $\eta_{\text {rel }}$ is relative viscosity of the polymer solution compared with the solvent, $\mathrm{C}$ is the concentration of the polymer solution (in $\mathrm{g} / \mathrm{dl}$ ), and $\kappa$ " is Kraemer's constant.

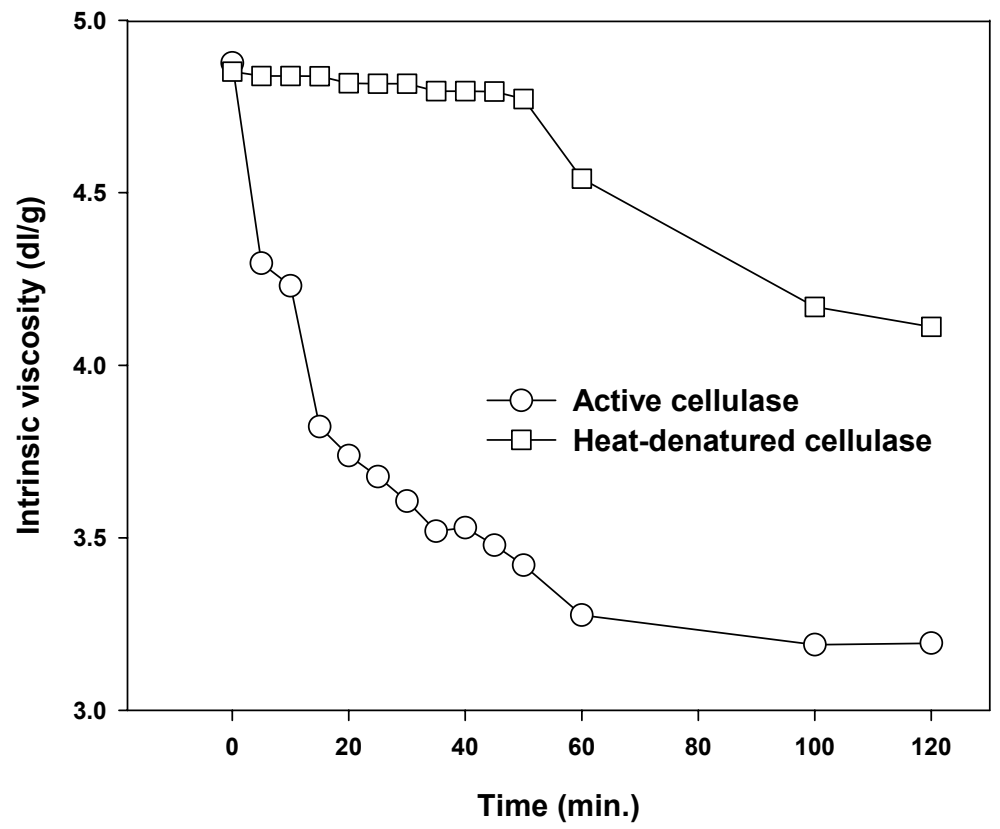

Fig. 4. Intrinsic viscosity of $\mathrm{CMC}$ with active and heat-denatured cellulase at $50{ }^{\circ} \mathrm{C}$ and $10 \mathrm{rpm}$.

Figure 4 shows the profiles of intrinsic viscosity of different concentrations of $7 \mathrm{M}$ $\mathrm{CMC}$ treated with active and heat-denatured cellulases for 2 hours at $50^{\circ} \mathrm{C}$ and a shear rate of $10 \mathrm{rpm}$. One observes a significant decrease in the intrinsic viscosity for both the heat-denatured and active enzymes. Thus, significant errors could occur in measuring the enzymatic activity by solution viscosity, if the polymeric effect is strong or varies from sample to sample.

To accommodate the polymeric effect, the solution viscosity can be adjusted. Assuming that the viscosity of a solution is related to friction between molecules within the solution and that the different components contributing to the change in solution viscosity are linear, then one can separate the decrease in viscosity of the active enzyme into two components. The first component is related to the decrease in the molecular weight of the CMC. The second component is related to the polymeric effect. The change in viscosity can be expressed as, 


$$
\left(\frac{d \eta}{d t}\right)=\left(\frac{d \eta}{d t}\right)_{M W}+\left(\frac{d \eta}{d t}\right)_{\text {polymer }}
$$

where the subscript $M W$ indicates the viscosity changes associated with changes in the molecular weight, and the subscript polymer indicates viscosity changes associated with polymeric effects. Equation 2 is not applicable when a large amount of enzymatic degradation has taken place, as the magnitude of the polymeric effect is influenced by the molecular weight, cf. Figure 5.
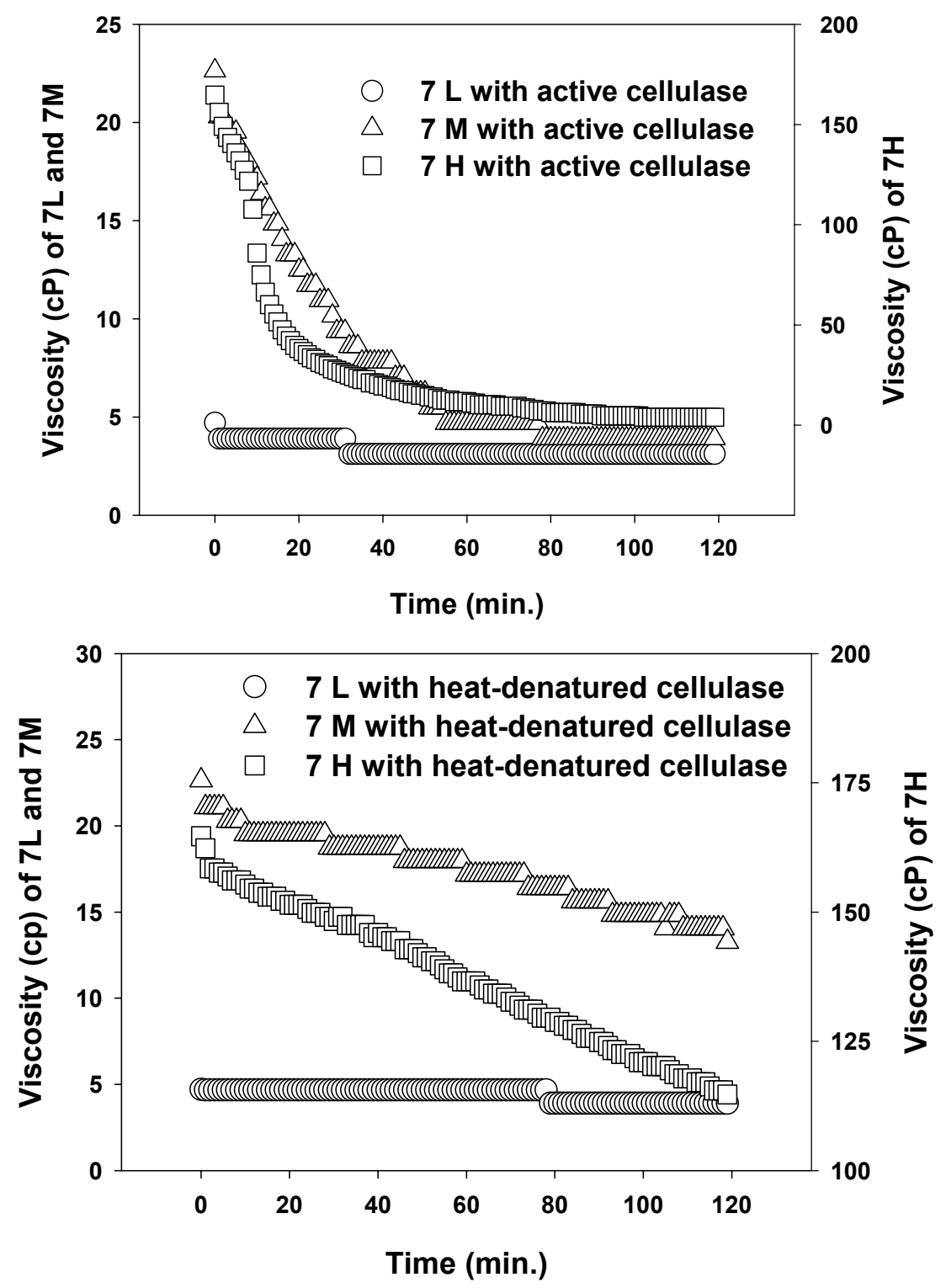

Fig. 5. Apparent viscosity of different molecular weights of CMC (1\% conc.) treated with active cellulase (top) and heat-denatured cellulase (bottom) at $50^{\circ} \mathrm{C}$ and $10 \mathrm{rpm}$ as a function of time. 
For relatively short degradation times, the change in viscosity as measured with the heat-denatured enzyme may be subtracted from the viscosity of the active enzyme solution to provide an estimate of the polymeric effect. For purposes of this estimation it is necessary to assume that the heat-denatured enzymes retain a molecular conformation that is not too different from that of the native enzymes. Using this modified viscosity data, a measurement of the change in viscosity attributed strictly to the catalytic activity can be made. Figure 6 shows the change in the viscosity adjusted for the polymeric effect compared to the unadjusted viscosity. As one can see in the figure, the polymeric effect is significant and should be compensated for in rheological measurements of enzyme activity. However, one also can see that this adjustment for the polymeric effect is nonsensical for large contact times, as the adjusted viscosity becomes negative. Thus, such a correction method should only be used for short contact times.

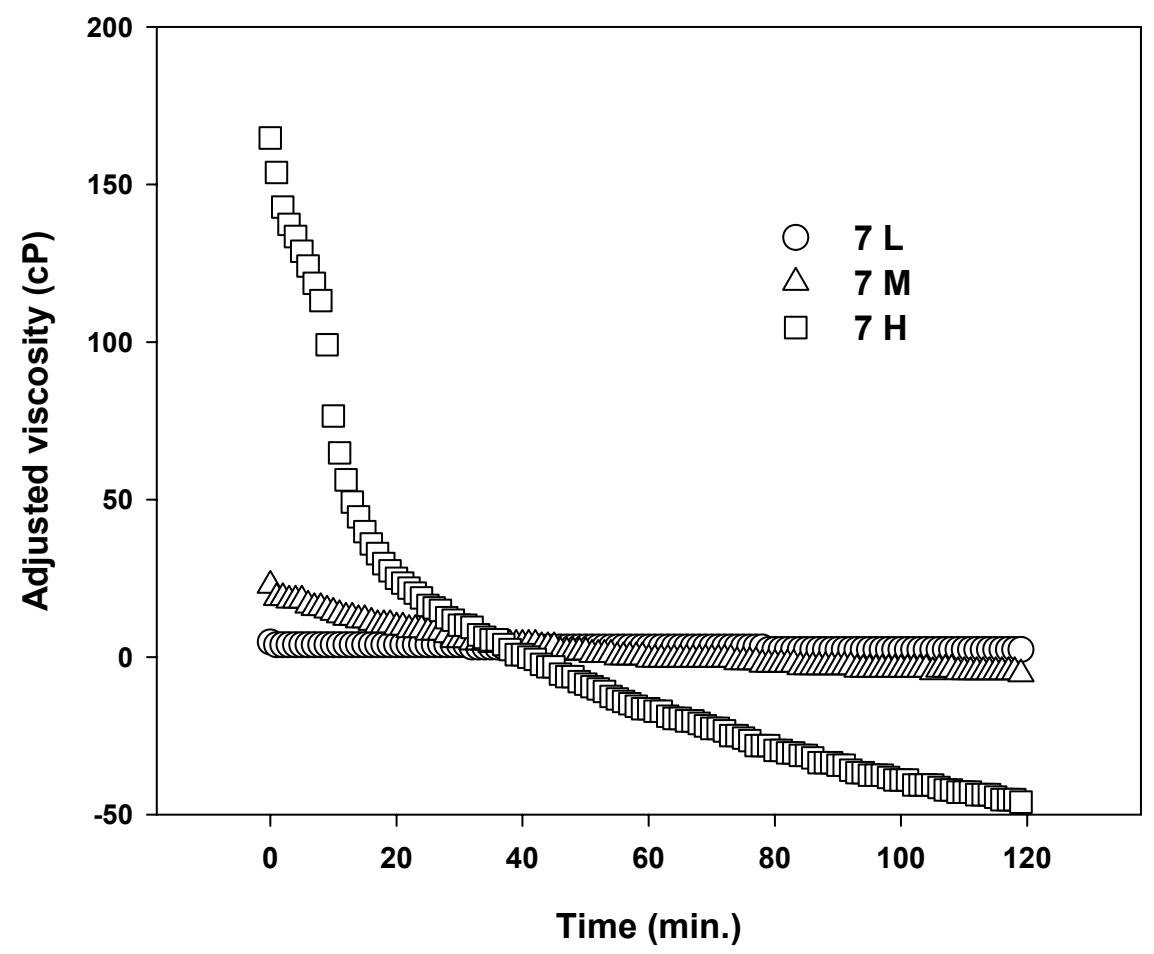

Fig. 6. Adjusted viscosities of the different molecular weights of CMC ( $1 \%$ conc.) as a function of time.

\section{Effect of Shear Rate on Viscosity}

Figure 7 shows the effect of shear rate on the measured viscosity of $1 \% 7 \mathrm{H} \mathrm{CMC}$ at $50{ }^{\circ} \mathrm{C}$ treated with a fixed enzyme dosage. A complex pattern of viscosity change is detected. A possible explanation for this complex pattern is the interaction of enzyme mixing and the effect of shear rate on the disruption of cellulase binding. At low shear rates, the enzymes must diffuse throughout the CMC solution with little assistance from mixing. This results in a slower drop in the viscosity of the CMC solution with time. As the shear rate increases, the mixing assistance becomes better at distributing the cellulase throughout the solution. This results in a more rapid decrease in the solution viscosity. 
However, at higher shear rates the viscosity does not decrease as fast as at intermediate shear rates. This may be attributed to the shear rate disrupting the cellulase/CMC binding and thus reducing the cellulase efficiency (Kaya et al. 1994; Kim et al. 1982). Thus, differences in shear rate can affect the measured viscosity change and the perceived cellulase activity.

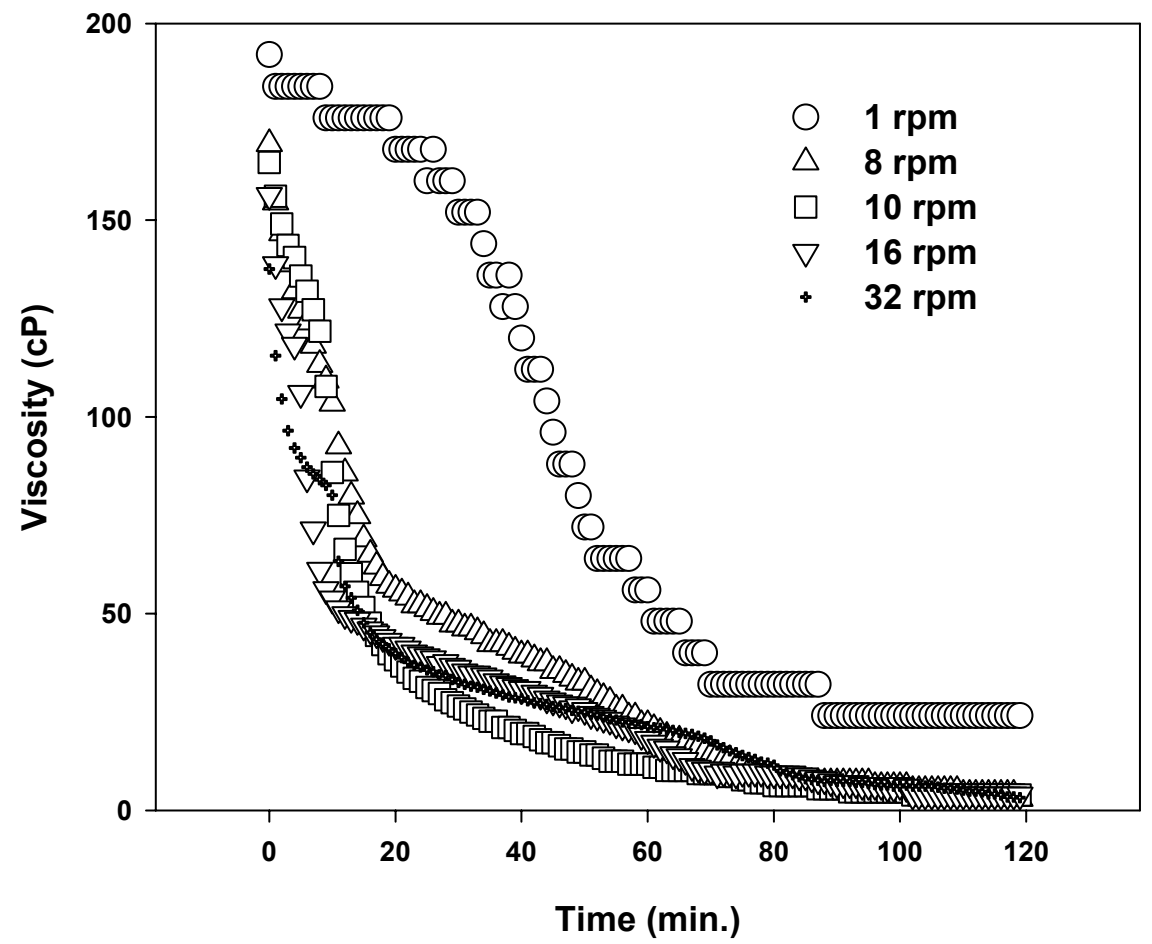

Fig. 7. Effect of shear rates in the range of 1 to $32 \mathrm{rpm}$ of $7 \mathrm{H} \mathrm{CMC} \mathrm{(1 \%} \mathrm{conc.)} \mathrm{treated} \mathrm{with}$ active cellulase as a function of reaction time.

\section{CONCLUSIONS}

The rheological behavior of anionic cellulose derivatives during the course of enzyme hydrolysis has been investigated. The effects of the active and heat-denatured cellulases, and a cationic polyelectrolyte (C-PAM) have been examined. Cellulose derivatives with different degrees of polymerization and substitution were examined. A polymeric effect, defined as a reduction in viscosity of the CMCs without significant formation of reducing sugars released from the degradation of the CMCs by enzymatic hydrolysis, has been observed in this study. The polymeric effect may be attributed to the interactions of the enzyme with the polymer in solution. This effect was observed with heat-denatured enzyme, active enzyme and high DS CMC (preventing enzymatic hydrolysis), and with the addition of C-PAM. The intrinsic viscosity, which may be related to the molecular weight of a dissolved material, is reduced by the polymeric effect. This may cause a significant error in the measurement of enzymatic activity with viscometric methods. Assuming a linear relationship between the changes in the solution 
viscosity attributed to enzyme hydrolysis and the polymeric effect, a means for correcting for the polymeric effect was proposed. This allows for making a correction for the polymeric effect during the onset of the hydrolysis reaction. Finally, the effect of shear rate on the viscosity change caused by enzymatic hydrolysis was examined. A complex behavior of viscosity change was found with shear rate. The results were interpreted to be a combination of improved mixing with higher shear rates and disruption in binding by higher shear rates.

\section{ACKNOWLEDGMENTS}

The authors acknowledge the McIntire-Stennis Program for its financial support of this research.

\section{REFERENCES CITED}

Almin, K. E., and Eriksson, K. E. (1967). "Enzymic degradation of polymers. I. Viscometric method for the determination of enzymic activity," Biochim. Biophys. Acta 139(2), 238-247.

Andreaus, J., Azevedo, H., and Cavaco-Paulo, A. (1999). "Effects of temperature on the cellulose binding ability of cellulases enzymes," J. Mol. Catal. B:Enzym. 7, 233-239.

Baker, J. O., Tatsumoto, K., Grohmann, K., Woodward, J., Wichert, J. M., Shoemaker, S. P., and Himmel, M. E. (1992). "Thermal denaturation of Trichoderma reesei cellulases studied by differential scanning calorimetry and tryptophan fluorescence," Appl. Biochem. Biotechnol. 34/35, 217-231.

Beguin, P., and Aubert, J.-P. (1994). "The biological degradation of cellulose," FEMS Microbiol. Rev. 13(1), 25-58.

Bhat, M. K. (2000). "Cellulases and related enzymes in biotechnology," Biotechnol. Adv. 18(5), 355-383.

Boisset, C., Armand, S., Drouillard, S., Chanzy, H., Driguez, H., Henrissat, B. (1998). "Structure-function relationships in cellulases: The enzymatic degradation of insoluble cellulose." in Carbohydrases from Trichoderma reesei and Other Microorganisms: Structures, Biochemistry, Genetics and Applications, M. Claeyssens, W. Nerinckx and K. Piens, eds., Royal Society of Chemistry, Cambridge, 124-132.

Britt, D. W., Jogikalmath, G., and Hlady, V. (2003). "Protein interactions with monolayers at the air-water interface," in Biopolymers at Interfaces, M. Malmsten, ed., Marcel Dekker, New York, ch. 16, 415-434.

Browning, B. L. (1967). "Viscosity and molecular weight." in Methods of Wood Chemistry, Vol. 2, B. L. Browning ed., Interscience Publishers, New York, ch.25, 519-557.

Doublier, J.-L., Garnier, C., Renard, D., and Sanchez, C. (2000). "Protein-polysaccharide interactions," Curr. Opin. Colloid Interface Sci. 5, 202-214.

Focher, B., Marzetti, A., Beltrame, P. L., and Carniti, P. (1991). "Structural features of cellulose and cellulose derivatives, and their effects on enzymatic hydrolysis," in 
Biosynthesis and Biodegradation of Cellulose, C. H. Haigler, ed., Marcel Dekker, New York, 293-310.

Ghose, T. K. (1987). "Measurement of cellulase activities," Pure Appl. Chem. 59(2), 257268.

Hoogendam, C. W., de Keizer, A., Cohen Stuart, M. A., Bijsterbosch, B. H., Smit, J. A. M., van Dijk, J. A. P. P., van der Horst, P. M., and Batelann, J. G. (1998).

"Persistence length of carboxymethyl cellulose as evaluated from size exclusion chromatography and potentiometric Titrations," Macromolecules 31, 6297-6309.

Kaya, F., Heitmann, J. A., and Joyce, T. A. (1994). "Cellulase binding to cellulose fibers in high shear fields," J. Biotechnol. 36, 1-10.

Kelly, R., Gudo, E. S., Mitchell, J. R., and Harding, S. E. (1994). "Some observations on the nature of heated mixtures of bovine serum albumin with an alginate and a pectin," Carbohydr. Polym. 23, 115-120.

Kim, M. H., Lee, S. B., and Ryu, D. D. Y. (1982). "Surface deactivation of cellulase and its prevention," Enzyme Microb. Technol. 4, 99-103.

Kraemer, E. O. (1938). "Molecular weights of celluloses and cellulose derivatives," Ind. Eng. Chem. 30, 1200-1203.

Miller G. L., Blum R., Glennon W. E., and Burton, A. L. (1960). "Measurement of carboxymethylcellulase activity," Anal. Biochem. 1, 127-132.

Mullings, R. (1985). "Measurement of saccharification by cellulases," Enzyme Microb. Technol. 7(12), 586-591.

Renard D., Boue F., and Lefebvre, J. (1997). "Protein-polysaccharide mixtures: Structure of the systems and the effect of shear studied by SANS," Physica B, 234-236, 289291.

Renard D., Boue F., and Lefebvre, J. (1998). "Solution and gelation properties of proteinpolysaccharide mixtures: signature by small angle neuron scattering and rheology," in Gums and Stabilizers for the Food Industry 9, E. Dickinson, and B. Bergenstabl, eds., Royal Society of Chemistry, Cambridge, 189-201.

Rouvinen, J., Bergfors, T., Teeri, T., Knowles, J. K. C., and Jones, T. A. (1990). “Threedimensional structure of cellobiohydrolase II from Trichoderma reesei," Science 249, 380-386.

Sharrock, K. R. (1988). "Cellulase assay methods: A review," J. Biochem. Bioph. Methods 17(2), 81-106.

Shimada, K., and Cheftel, J. C. (1989). "Sulfhydryl group/Disulfide bond interchange reactions during heat-induced gelation of whey protein isolate," J. Agric. Food. Chem. 37,161-168.

Tuinier R., Dhont J. K. G., and de Kruif C. G. (2000). “Depletion-induced phase separation of aggregated whey protein colloids by an exocellular polysaccharide," Langmuir 16, 1497-1507.

Vlasenko, E. Y., Ryan, A. I., Shoemaker, C. F., and Shoemaker, S. P. (1998). "The use of capillary viscometry, reducing end-group analysis, and size exclusion chromatography combined with multi-angle laser light scattering to characterize endo-1,4- $\beta$-D-glucanases on carboxymethylcellulose: A comparative evaluation of the three methods," Enzyme Microb. Technol. 23(6), 350-359. 
Wu, B., Zhao, Y., and Gao, P. J. (2006). "A new approach to measurement of saccharifying capacities of crude cellulase," BioResources 1(2), 189-200.

Zhou, X., Chen, H., and Li, Z. (2004). "CMCase activity assay as a method for cellulase adsorption analysis,” Enzyme Microb. Technol. 35(5), 455-459.

Article submitted: October 23, 2006; First review cycle completed November 20, 2006;

Revision accepted: Dec. 21, 2006; Article published: January 2, 2007. 